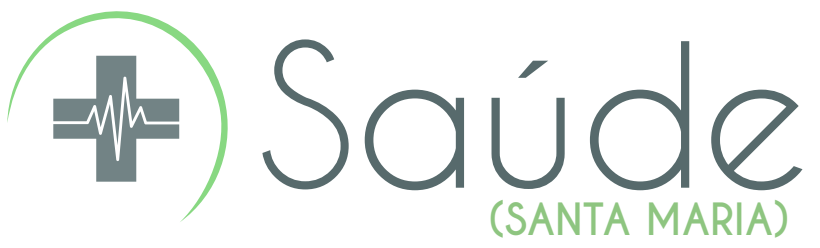

Relato de Experiencia

DOI: $10.5902 / 2236583424084$

Como citar este artigo:

Monteiro BR, Ataíde CAV, Silva CJA, Neres JNS, Medeiros ER, Simpson CA. Educação em saúde para a hanseníase: experiência da enfermagem. Revista Saúde (Sta. Maria). 2018; 44(1):1-5

Autor correspondente:

Bruna Rodrigues Monteiro

E-mail: beatriz_luk@hotmail.com

Telefone: (81) 99838-6845

Formação Profissional: Enfermeira.

Residente em Saúde da Mulher na Residencia Multiprofissional em Saúde da Universidade Federal do Pernambuco. Recife, Pernambuco, Brasil.

Filiação Institucional: Universidade Federal do Pernambuco (UFPE)

Endereço para correspondência:

Rua Visconde de Barbacena, 329,

Edifício San Bernard, Apartamento 201, Várzea, Recife, Pernambuco,

Brasil. CEP: 50740-445.

Data de Submissão: 20/09/2016

Data de aceite: 20/03/20/8

Conflito de Interesse: Não há

conflito de interesse

\section{Educação em saúde para a hanseníase: experiência da enfermagem}

\section{Healtheducation for leprosy: nursing experience}

\author{
Bruna Rodrigues Monteiro, Cáthia Alessandra Varela Ataíde, Carlos Jor- \\ dão de Assis Silva, Jéssica Naiara Silva Neres, Eliabe Rodrigues de Medei- \\ ros, Clélia Albino Simpson
}

\section{RESUMO}

\begin{abstract}
A Hanseníase é uma doença infecto-contagiosa, com diagnóstico tardio e que evolui para lesões cutâneas reduzindo a sensibilidade térmica, dolorosa e tátil, nesse sentido o Ministério da Saúde, retrata a importância das atividades educativas na área da saúde como um ponto estratégico para seu controle. Tem-se como objetivo relatar a experiência de discentes do curso de graduação e pós-graduação em enfermagem no desenvolvimento de atividades de educação em saúde sobre a hanseníase. Estudo descritivo do tipo relato de experiência sobre educação em saúde em hanseníase, desenvolvido por pós-graduandos e graduandos em enfermagem em um evento científico. Os dados foram organizados nas seguintes categorias: Conceito geral da hanseníase e seu aspecto histórico; Sinais e Sintomas; Diagnóstico e Tratamento; Dinâmica Interativa. É perceptível a interação dos discentes de enfermagem no desenvolvimento contínuo da educação em saúde, conciliando o conhecimento cientifico junto ao conhecimento empírico, contribuindo assim na eliminação da Hanseníase.
\end{abstract}

Descritores: Educação em Saúde; Hanseníase; Enfermagem.

\begin{abstract}
Leprosy is an infectious disease with late diagnosis and evolves into skin lesions reducing thermal, painful and tactile sensitivity in this regard the Ministry of Health, portrays the importance of educational activities in the health sector as a strategic point for your control. It has been as objective report the experience of undergraduate course students and nursing graduate in the development of health education activities about leprosy. Method: A descriptive study of type experience report on health education in leprosy, developed by graduate and undergraduate students in nursing in a scientific event. Data were organized in the following categories: general concept of leprosy and its historical aspect; Signals and symptons; Diagnosis and treatment; Interactive dynamics. It is noticeable interaction of nursing students in the continuing development of health education, combining scientific knowledge with the empirical knowledge, thus contributing to the elimination of leprosy.
\end{abstract}

Descriptors: Health Education; Leprosy; Nursing. 


\section{Introdução}

A Hanseníase é uma doença infecto-contagiosa causada pelo Mycobacterium leprae, diagnosticada tardiamente e que evolui para lesões cutâneas reduzindo a sensibilidade térmica, dolorosa e tátil. ${ }^{1}$

A Organização Mundial da Saúde (OMS) formulou estratégias para vencer os constantes desafios na área de Saúde, onde um deles é a constante presença da Hanseníase, formulando assim uma estratégia global de Redução Adicional da Carga da Hanseníase: 2011-2015, pelos países endêmicos. ${ }^{2}$

Dentre esses países, encontra-se o Brasil com coeficiente de prevalência de 1,51 caso de hanseníase para 10 mil habitantes no ano de 2012. No cumprimento da meta proposta pela OMS, o país tem como função: reduzir a taxa de casos novos de hanseníase e realizar o diagnóstico precoce, favorecendo assim a redução de novos casos e, posteriormente, reduzindo a transmissão da doença na sociedade. ${ }^{3}$

Nessa perspectiva o Caderno de Atenção Básica, formulado pelo Ministério da Saúde, retrata a importância das atividades educativas na área da saúde como um ponto estratégico na eliminação da doença. ${ }^{4}$

A educação em saúde é uma prática de preservação da saúde individual e coletiva. Ao tomar como objeto de reflexão, ela representa a melhoria da qualidade de vida, despertando a necessidade do indivíduo em adotar medidas relacionadas ao bem-estar físico, social e mental. ${ }^{4}$

Concebe-se a hanseníase como um importante problema de saúde pública, merecedora de destaque por parte das políticas públicas que promovam maior conscientização aos profissionais inseridos nos serviços de saúde, sobre a importância das ações de educação com a população. Nestas condições, os usuários poderão compreender o que é a hanseníase e suas formas de detecção precoce, o que pode favorecer melhores condições de vida e de cuidado com a saúde. $^{5}$

Assim, a educação em saúde figura como uma importante oportunidade de atuação do Enfermeiro, no sentido de promover a saúde e prevenir agravos, além de acarretar na autonomia dos usuários. Entretanto, é importante ressaltar que estas ações devem ser operacionalizadas de modo horizontalizado com base na escuta e no relacionamento humanizado, superando a mera transmissão dos conteúdos. ${ }^{6}$

Destaca-se que nos últimos anos a divulgação dos sinais e sintomas da hanseníase pelos diversos meios de comunicação contribui com o aumento na detecção e no número absoluto dos casos de hanseníase no Brasil. ${ }^{7}$

Desta forma, objetivou-se relatar a participação de acadêmicos do curso de graduação em Enfermagem na realização de uma ação de educação em saúde sobre Hanseníase.

\section{Metodologia}

Estudo descritivo, de abordagem qualitativa, do tipo relato de experiência de atividade sobre educação em saúde, vivenciada por graduandos e pós-graduandos do curso de graduação em Enfermagem da Universidade Federal do Rio Grande do Norte (UFRN), durante a XXI Semana de Ciência, Tecnologia e Cultura da UFRN (CIENTEC), realizada no ano de 2014, no Município de Natal, capital do Estado do Rio Grande do Norte, Brasil.

A CIENTEC é um evento da Universidade Federal do Rio Grande do Norte que acontece todos os anos, expondo os principais fundamentos das atividades científicas, tecnológicas e culturais da Universidade, buscando, dessa forma, uma interface com a sociedade. A Universidade, enquanto espaço da troca de conhecimento e do diálogo de ideias, organiza a Semana de Ciência e Tecnologia da UFRN a partir de pavilhões que abrigarão exposições interativas e transdisciplinares, enfocando as produções dos Centros Acadêmicos e dos Órgãos Especializados e Suplementares e dos Eventos Acadêmicos, como congressos, seminários e exposição de pôsteres.

A ação aconteceu por ocasião da XXI CIENTEC. A atividade foi planejada em dois momentos distintos. No primeiro momento, foi realizada uma capacitação, diante a necessidade dos acadêmicos, sobre hanseníase e vigilância em saúde, desenvolvida pelos membros do grupo de pesquisa: Ações promocionais e de atenção a grupos humanos em Saúde Mental e Saúde Coletiva, a qual os mesmos fazem parte.

O segundo momento faz referência aos estandes na XXI CIENTEC, os quais foram planejados painéis cronológicos, baseado no álbum seriado elaborado pela Fundação Paulista contra a Hanseníase de 2015, retratando assim o aspecto histórico, conceito da doença, sinais e sintomas, como é feito o diagnóstico, tratamento e prevenção. A abordagem foi 
feita pelos discentes que participaram da capacitação aos visitantes da exposição, que em sua maioria eram alunos do ensino médio e fundamental das escolas de Natal e do RN, além de alunos de graduação de todos os cursos.

Ainda nessa dimensão, destaca-se que a atividade é observada como uma atividade essencial na qualificação do aluno universitário, que estão apoiados em três eixos: ensino, pesquisa e extensão. Esta última quando relacionada à saúde coletiva desempenha atividades pertinentes de reorientação da saúde. ${ }^{8}$

\section{RESULTADOS:}

A partir exposição do estande durante os dias do evento, o público que visitava CIENTEC iniciou a sua participação pelas estações criadas ao longo do estande. As informações foram organizadas em quatro estações específicas:

\section{Conceito geral da Hanseníase e seu aspecto histórico}

O trajeto do estande inicia com o seguinte questionamento ao público: "O que é hanseníase?". Esse questionamento objetivava estimular o público a demonstrar seus conhecimentos prévios sobre a Hanseníase. Posteriormente iniciouse a explanação da questão histórica da doença, abordando as questões bíblicas, o estigma, aspectos históricos e os leprosários existentes no Brasil e no Estado do Rio Grande do Norte, encerrando com a lei que oficializou a mudança da nomenclatura de lepra para hanseníase.

\section{Sinais e Sintomas}

Como forma de representar os sinais e sintomas da doença, os discentes criaram a representação estrutural do corpo humano, colocando uma variedade de patologias que atingem diretamente a pele, tendo também como manifestações clínicas o aparecimento de manchas, dentre elas, a hanseníase, assim foi explicando ao ouvinte que nem toda mancha na pele está diretamente ligada a doença, apresentando assim as características peculiares da doença.

\section{Diagnóstico e Tratamento}

Relacionado ao diagnóstico os ouvintes visualizaram os principais testes de sensibilidade para a confirmação da doença, como por exemplo, o teste de sensibilidade tátil e térmica, sendo explicados quanto à função de cada um. Posteriormente foi explanado sobre o tratamento, como é realizado, a base de que fármacos, durante quanto tempo, se o mesmo é oferecido pelo Sistema Único de Saúde, finalizando assim essa estação.

\section{Dinâmica Interativa}

Finalizando o circuito foi realizado uma dinâmica interativa, sendo um de tabuleiro com perguntas e respostas, objetivando-se conduzir os participantes a uma pequena retrospectiva sobre 0 assunto abordado, ressaltando os principais conceitos e informações relatados anteriormente. Após o término do jogo os participantes registravam sua presença através de mensagens e suas assinaturas em um flip-chart com o título "deixe sua marca".

\section{Discussão}

Responsável por formar indivíduos generalistas e reflexivos, o curso de graduação em enfermagem tem como função capacitar o discente no desenvolvimento de atividades voltadas para comunicação, atenção à saúde em todos os níveis, tomada de decisões, administração e gerenciamento, bem como a realização da educação permanente, a qual possibilita os discentes a construção do conhecimento por meio de ações desenvolvidas.

Dentre elas, existe a educação em saúde, processo que requer uma aproximação ativa com público. Como facilitador dessa atividade alguns instrumentos contribuem no desenvolvendo das intervenções, como os recursos audiovisuais, as dinâmicas, imagens e a necessidade de uma linguagem comum ao ouvinte, promovendo de forma efetiva a intervenção e ao mesmo tempo atendendo o objetivo proposto pela atividade..$^{10}$

Durante a abordagem inicial ao público através da pergunta exposta na forma de álbum seriado sobre "o que é a Hanseníase?", os participantes relatavam ausência de conhecimento, alguns informavam que a doença era caracterizada por manchas na pele. Nesse momento, houve a explicação sobre a etiologia da hanseníase e seus meios de transmissão. Ao informarmos que a hanseníase foi denominada por muitos anos como "lepra" os participantes, principalmente adultos 
e idosos, associaram a doença com questões bíblicas, informando ser uma doença considerada no passado incurável, deformante e associada ao pecado. Ao relatarmos sobre as instituições destinadas ao exílio e tratamento dos pacientes com hanseníase alguns ouvintes informaram que estiveram presentes na colônia durante esse período, visualizando pessoas isoladas em seu recinto e com lesões cutâneas pela pele oriundas da doença.

Durante todas as etapas os discentes buscaram considerar o conhecimento pré-existente dos participantes, suas experiências e impressões relacionados a hanseníase, uma vez que o profissional de saúde quando presente na atividade educativa necessita reconhecer o saber do público-alvo, ocupando papel de mediador do processo de compartilhamento sem impor os conhecimentos científicos, possibilitando assim o diálogo entre ambos. ${ }^{11}$

A realização do jogo com as perguntas e resposta foi crucial para a avaliação dos conhecimentos aprimorados dos ouvintes sobre a hanseníase, sendo evidenciado pelas respostas dadas pelos participantes, sendo percebido que eles compreenderam o que é a hanseníase, seus aspectos históricos, sinais e sintomas, modo de transmissão, tratamento e principalmente como ela pode ser diagnosticada e como o indivíduo com a doença pode conviver de maneira saudável com toda a sociedade quando se inicia o tratamento e também após o seu término.

A educação em saúde, quando entendida pelo público-alvo contribui no desenvolvimento do diagnóstico precoce da hanseníase, bem como auxilia na prevenção de incapacidades trazidas pela doença, atingindo, assim, as metas estabelecidas pelo Programa Nacional de Controle de Hanseníase (PNCH) ${ }^{12,13}$ Essa prática apresenta como percussor principal o enfermeiro, profissional que busca o atendimento integral do indivíduo, por meio da promoção e prevenção da saúde. ${ }^{14}$

Como principal desafio da educação em saúde se tem a necessidade de saber se as informações transmitidas ao ouvinte são adequadas para o mesmo, ao ponto de ser aceita na sua vida diária, buscando em si a manutenção da saúde. Porém a receptividade e a interação do público com o profissional pode ser caracterizada como uma espécie de avaliação positiva capaz de estimular o desenvolvimento de ações de autocuidado com si e com o próximo.15

\section{Conclusão}

Através do estudo, observa-se que as práticas de educação em saúde adotadas na realização do estande foram atrativas para o público, bem como gerou nos estudantes de graduação a necessidade de saber conciliar o conhecimento acadêmico com o conhecimento de cada indivíduo, havendo interação de ambas as partes e assim contribuindo para desmistificação e erradicação da hanseníase.

Contudo as ações de educação em saúde devem ocorrer de forma interativa, auxiliando no entendimento do público presente, visto que estes também são protagonistas do saber, e disseminadores de informações entre seus familiares e amigos.

Assim, fica evidente a importância da educação em saúde como prática constante da vida acadêmica, isto porque como futuros profissionais da enfermagem, os alunos de graduação devem ter conhecimentos e experiências em áreas de ensino, já que a enfermagem configura-se como uma profissão que tem como característica a possibilidade de disseminar informação em saúde para uma parcela significativa da população. E cabe à academia promover e garantir esse espaço de aprendizado aos seus alunos. Eventos como a CIENTEC contribuem para a promoção desses espaços e dão a oportunidade dos alunos se tornarem partícipes desse processo de ensino-aprendizagem.

Vale ressaltar que as atividades educativas não competem apenas ao acadêmico durante a sua formação, pertence também ao profissional de saúde que tem o contato diário com o cliente. Necessitando rever o papel dos profissionais de saúde já formados, os quais necessitam adotar uma metodologia que possibilite esse vínculo de educador e aprendiz, além da necessidade de atuação de outros profissionais de forma a possibilitar a educação em saúde como um campo interdisciplinar.

\section{Referências}

1. Alves CJM, Barreto JA, Fogagnolo L, Contin LA, Nassif PW. Avaliação do grau de incapacidade dos pacientes com diagnóstico de hanseníase em Serviço de Dermatologia do Estado de São Paulo. Rev Soc Bras Med Trop. 2010;43(4):460-1.

2. Organização Mundial da Saúde. Estratégia Global Aprimorada para Redução Adicional da Carga da 
Hanseníase (2011-2015). Brasília: Organização Pan-Americana da Saúde; 2010.

3. Alberts CJ, Smith WCS, Meima A, Wang L, Richardus JH. Potential effect of the World Health Organization's 2011-2015 global leprosy strategy on the prevalence of grade 2 disability: a trend analysis. Bull World Health Organ. 2011;89:487-95.

4. Marinus MWLC, Pacheco HF, Lima FT. Saúde do escolar: uma abordagem educativa sobre Hanseníase. Sau \& Transf Soc. 2012;3(1):72-8.

5. Pinheiro MGC, Medeiros IBG, Monteiro AI Simpson CA. O enfermeiro e a temática da hanseníase no contexto escolar: relato de experiência. Rev pesqui cuid fundam (Online). 2015;7(3):2774-80.

6. Roecker S, Budó MLD, Marcon SS. Trabalho educativo do enfermeiro na estratégia saúde da família: dificuldades e perspectivas de mudanças. Rev Esc Enferm USP. 2012;46(3):641-9.

7. Arantes CK, Garcia MLR, Filipe MS, Nardi SMT, Paschoal VDA. Avaliação dos serviços de saúde em relação ao diagnóstico precoce da hanseníase. Epidemiol Serv Saúde. 2010;19(2):155-64.

8. Silva SS, Bonfim ES, Silva MA, Souza NS. Agente comunitário de saúde: o uso da educação em saúde como facilitadora do cuidado. Em Extensão 2014;13(2):122-8.

9. Brasil. Ministério da Educação. Conselho Nacional de Educação. Câmara de Educação Superior. Diretrizes Curriculares Nacionais dos Cursos de Graduação em Enfermagem, Medicina e Nutrição. Brasília: Ministério da Educação; 2011.

10. Fonseca JMA, Radmann CS, Silva DRC, Guimarães AEV, Oliveira ME. Contribuições da fisioterapia para educação em saúde e grupo de autocuidado em hanseníase: relato de experiência. Revista Gestão \& Saúde. 2015; 6(1): 770-77.

11. Colome JS, Oliveira DLLC. Educação em saúde: por quem e para quem? A visão de estudantes de graduação em enfermagem. Texto contexto- enferm. 2012;21(1):177-84.

12. Ministério da Saúde (Brasil). Boletim Epidemiológico da Hanseníase no Brasil. 2013; 44(11).

13. Sousa BRM, Moraes FHA, Andrade JS, Lobo ES, Macedo EA, Pires CAA et al. Educação em saúde e busca ativa de casos de hanseníase em uma escola pública em Ananindeua, Pará, Brasil. Sousa (2013). Rev Bras Med Fam Comunidade. 2013; 8(27): 143-9.

14. Nascimento GRC, Barrêto AJR, Brandão GCG, Tavares CM. Ações do enfermeiro no controle da hanseníase. Rev eletrônica enferm. 2011; 13(4):743-50.

15. Silva MCD, Paz EPA. Educação em saúde no programa de controle da hanseníase: a vivência da equipe multiprofissional. Esc Anna Nery. 2010;14(2):223-29. 